ble in many of the chapters. He was a member of the Avoiding Nuclear War Project out of which the book grew.

The final list of recommendations is divided into ten sections. Two of the sections are hawkish, two are dovish and six are owlish. These proportions reflect the editors' belief that at present the dangers of war through weakness or paranoia are over-rated and the dangers of war through muddle are under-rated. The majority of their recommendations are directed toward avoiding a nuclear version of the outbreak of war in 1914, an outbreak driven by geographical and historical accident rather than by any grand design.

A small sample may indicate the general flavour of the editors' recommendations. I choose five that I consider characteristic, one hawkish, "Don't adopt a NoFirst-Use policy." one dovish, "Don't assume that nuclear deterrence will last forever," and three owlish, "Do reduce

\section{Manual works}

\section{Graham Richards}

A Handbook of Computational Chemistry: A Practical Guide to Chemical Structure and Energy Calculations. By Tim Clark. Wiley: 1985. Pp.332. £35.80, \$35.

Semi-Empirical Methods of Quantum Chemistry. By Joanna Sadlej. Ellis Horwood: 1985. Pp.386. £47.50, \$71.25.

COMPUTATIONAL chemistry has come out of the closet. No longer is the calculation of molecular conformational energy or electronic distribution confined to a small clique of tolerated but mildly deviant academics who scorn conventional experimental practice. Even in the staid halls of industry, theoreticians are now to be found working alongside straight organic chemists, designing novel compounds with the aid of calculations - both quantum mechanical and of the molecular mechanics type - and, increasingly, assisted by sophisticated computer graphics displays

This growing army has a real need for a handbook which details not the theory behind the methods used, but rather where to start and how to use the program packages available. The most favoured weapons currently in use for elucidating conformation are programs based on molecular mechanics (treating molecules as balls and springs with empirical force fields). These are supplemented with molecular orbital calculations to unravel the electronic details, with the semiempirical methods known by the acronymic titles MINDO, MNDO and MOPAC becoming standard, together with variants on the $a b$ initio molecular orbital method developed by Pople and his co-workers (currently known as GAUSSIAN 82).

The use of all these packages requires reliance on short-range theater nuclear weapons, do work with the Soviets to prevent and manage crisis, do encourage nongovernmental contact with the Soviets." It is not necessary to agree entirely in order to applaud the clarity of their thinking and the brevity of their style.

I happen to disagree strongly with their No-First-Use recommendation, and still I recommend their book whole-heartedly as a fair and lucid statement of the nuclear dilemma. Only one essential thing is absent from their world-view. They lack a sense of the absurd. It may be that a resolution of the nuclear dilemma will come in the end from a general recognition of its absurdity. I would like to add one more recommendation to their list, "Don't take nuclear weapons more seriously than they deserve."

Freeman J. Dyson is in the Institute for $A d v-$ anced Study, South Olden Lane, Princeton, New Jersey 08540, USA.

the chemist to present as input the geometrical structure of the molecule under consideration. Clark's excellent manual provides clear and detailed instructions on how to submit data for the most widely adopted packages. It is replete with examples of sample input and of output, just the thing to enable an experimental chemist to be able to use the available programs. The book does not provide much about the general background theory, nor does it underline the rather shaky theoretical basis for some of the methods (these matters are, however, discussed in many, more conventional textbooks on theoretical chemistry). Instead we have a very practical manual, the review copy of which has already been tested by novice graduate students with happy results. The theoretical section of chemistry bookshelves is generally overstocked, but this book fills a genuine gap and will undoubtedly be found at the elbow of many users of packages.

Sadlej's text is in an overlapping area and is a more conventional account of the semi-empirical methods of quantum chemistry: sadly it is the book one wanted ten years ago. The Polish edition was written in 1976 and although "revised", the age shows. The bibliography goes up only to 1978, and the techniques upon which the book concentrates - the Hückel method and CNDO - are both now somewhat passé.

Those theoretical chemists who resent the loss of their exclusive and even furtive world will no doubt grumble that running a computer package no more makes one a theoretician than sleeping in a garage makes one a mechanic. But computational chemistry is on the rise and life should be made easier for the virgins.

Graham Richards is a lecturer in the Department of Physical Chemistry, University of Oxford. South Parks Road, Oxford OXI $3 Q Z, U K$

\section{Cool side of life}

\section{Pierre Douzou}

Biophysics and Biochemistry at Low Temperatures. By Felix Franks. Cambridge University Press:1985. Pp.210. £25, $\$ 44.50$.

Although Felix Franks in a sense is correct in his forward "cold is the fiercest enemy of many forms of life", biologists dream of exploiting it for the cryopreservation of "spare parts" of living systems amongst other problems. Like physicists some decades ago, biologists are becoming increasingly interested in the measurement, interpretation and exploitation of natural phenomena at low temperatures, and cryogenic research is slowly becoming a branch of this discipline. Franks' book is a step along the way in this endeavour.

The book gives clear answers to the classical questions about the physics and physical chemistry of water at sub-zero temperatures, the effects of its behaviour on life, and current applications in biochemistry. Other specialized chapters survey our understanding of the responses of the cell and of living organisms to cold, and of the problems of their preservation in the laboratory, a problem still dominated by empiricism and clouded by the interplay of an extraordinary variety of factors. The book ends with a survey of future prospects in this challenging but promising field.

Franks has been a leading contributor to research on water structure and properties, here he combines this expert insight with his interest and recent experience in cryopreservation for the task of explaining what is known and what is uncertain, and of suggesting what can be done to set up a useful cryobiology. The writing of a book on this topic demands a grasp of many specialized fields and is a daunting task, but the result here is agreeable and very instructive, because the book reveals its origin as a lecture course by a man trained as a physical scientist who drifted into the life sciences "fairly late in life and [who] never received any formal teaching in biological dogma" and had first to teach himself.

The book gives a strong impression of unity, carries with it a tone of optimism and in some cases of certitude and is valuable in that it covers a range of subject material which has never before been brought together in such an organized and concise yet clear manner. It will be of great interest to that growing band of researchers working on the cryobehaviour of living organisms.

Pierre Douzou is Professor at the Museum d'Histoire Naturelle, Paris and head of Department at the Institut de Biologie Physicochimique, 13 rue P.M. Curie, 75005 Paris, France. 